\title{
Mathematics and music: the architecture of nature
}

\author{
F. Morandi ${ }^{1}$, E. B. P. Tiezzi ${ }^{2}$ \& R. M. Pulselli ${ }^{1}$ \\ ${ }^{1}$ Department of Chemistry, University of Siena, Italy \\ ${ }^{2}$ Department of Mathematical Sciences "Roberto Magari", \\ University of Siena, Italy
}

\begin{abstract}
The link between mathematics and music has ancient origins and, over the centuries, it has been adding more and more new content. Mathematics and music are two languages apparently very different but in reality they are the two universal languages: numbers and notes are used worldwide as a global language. In this work we see how math and music provide the keys for understanding the wonderful book of Nature. We discuss in particular the "design of the cosmos" as proposed by Kepler in the Harmonice mundi: the German astronomer's survey on the architecture of the cosmos is based, in fact, on geometry, with reference to static aspects, and on music, with reference to kinematics. This theoretical framework represents not only the link between the astronomical revolution and the scientific revolution of the seventeenth century, but also the central point for the definition of the Newton's law of universal gravitation.
\end{abstract}

Keywords: mathematics, music, design.

\section{Introduction}

The investigation on the origin of the universe and the structure of nature has always been a highly discussed research topic. Pythagoras (575-475 BC) was the first to identify in mathematics and music the only two languages that can explain the architecture of nature [2, 4, 7]. A description of the natural world through the geometry is found in the Plato's Timaeus (360 BC) [9]: in which five regular polyhedra (known as the "Platonic solids") interact with the elements of Empedocles. This was the starting point for Kepler's research (1571-1630) on 
the design of the cosmos. In his works, especially in the Harmonice mundi, Kepler made mathematics, music, astronomy and astrology relate to each other. Through the geometry, he identified musical intervals and their relationship with the orbits of the planets and provided the famous Third Law from which, subsequently, Newton (1643-1727) deduced his Law of Gravitation.

\section{From Pythagoras to Kepler}

Pythagoras was the first philosopher to deal with the description of nature: he considered numbers at the origin of everything. In particular, the number that gives rise to the universe is the number ten $(10=1+2+3+4)$, the tetractys, and the figure represented by it, an equilateral triangle.

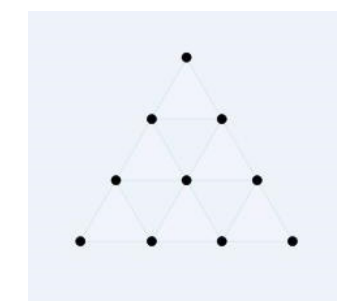

Figure 1: Pythagoric tetractys.

The Pythagoreans described the universe and natural phenomena by means of mathematics: they were always looking for proportions and harmonies regularities in natural phenomena.

In his Manual of Harmony, Nicomachus (a Greek mathematician of the first century AD) explained how Pythagoras discovered the numerical relations between the sounds produced by two pinched strings: Pythagoras, making various experiments on the monochord, determined the ratios of the lengths of two strings so that, when they struck, produce consonant sounds. Vincenzo Galilei, Kepler and Newton, in addition to music theorists of the Middle Ages, refer to this discovery.

In the Timaeus Plato referred to the Pythagorean doctrine [9]: the Demiurge is an almost divine "craftsman" which gives an order to the original chaos following a mathematical structure. In his theory, the basic structure of the universe consists of the five Platonic solids, namely the five regular convex polyhedra (tetrahedron, cube, octahedron, dodecahedron, icosahedron), which are put in correspondence with the elements of Empedocles: tetrahedron $\rightarrow$ fire, octahedron $\rightarrow$ air, cube $\rightarrow$ land, icosahedron $\rightarrow$ water. The dodecahedron has a special role because it represents the whole universe.

The construction of the five Platonic solids is found for the first time in Euclid's Elements [11], in the thirteenth book, and this construction was then elaborated by Kepler in his design of the world.

All Kepler's work concerns the description of nature [5, 6, 8]. The German astronomer aimed to provide the key for understanding the book of nature through mathematics and music. We find the geometric-harmonic vision of the 

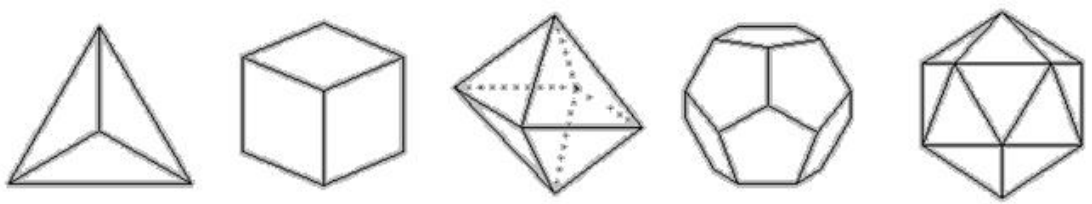

Figure 2: Platonics solids: tetrahedron, cube, octahedron, dodecahedron, icosahedron.

cosmos by Kepler in three of his books: Mysterium cosmographicum, Astronomia nova and Harmonice mundi. In Mysterium cosmographicum [6] he described the benefits of the Copernican theory compared to the Ptolemaic theory. He presented his geometric vision of the universe showing that distances between the Sun and the six planets can be determined by the five regular solids, inscribing and circumscribing a sphere to each of them. See fig. 3 .

Based on Platonic solids inscribed within concentric spheres, the Kepler's system explained the number and size of planets and we see that the orbits are not circular but eccentric curves. Kepler also puts his model in relation to music by relating not only the number of planets to the basic musical intervals,

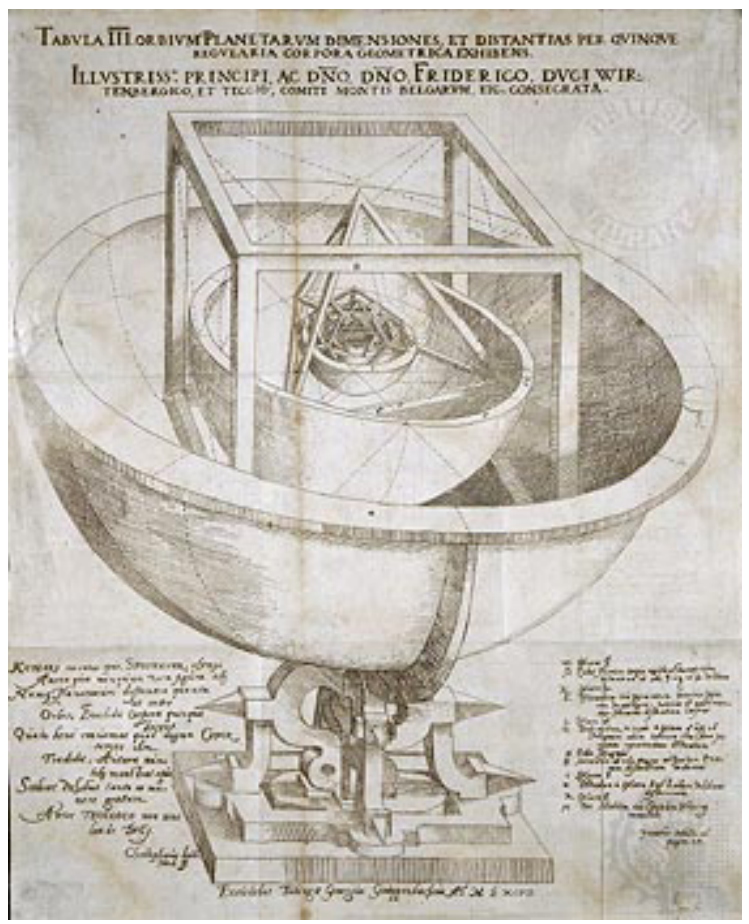

Figure 3: Model of the solar system in the Mysterium cosmographicum. 
identified by Pythagoras, but also the ratio of the distances of planets from the sun with the ratio at which these intervals are indicated. The astronomer, however, realized that his model, that is in movement in time and not static like the Aristotelian-Ptolemaic system, is not yet a true mirror of nature but a merely tool to make calculations. He continues his research in the Astronomia nova, born from the meeting with Tycho Brahe, in which he described the first two laws of planetary motion:

- First Law (1608): the planet's orbit is an ellipse with the Sun in one focus;

- Second Law (1609): the radius vector, that joins the center of the Sun with the center of the planet, describes equal areas in equal times.

The Harmonice Mundi [1, 5], conceived in 1599 as a result of Misterium cosmographicum and not completed until 1916, is a work in five books in which Kepler built a theory to explain the world and the nature based on geometry, that is the instrument that God used for the creation and that man uses for his work. The five books deals with geometry, arithmetic, music, astrology and astronomy. The order in which the author wrote them shows us the importance of music for the design of the universe: the first to be written was the third (about music), followed by the fourth and fifth, while the first and second were conceived later as necessary for understanding the other three. Kepler, although he accepted as a starting point the music theory of Zarlino (which refers to the Pythagorean), constructed a theory of music based on the regular polygons drawn with ruler and compass. He argued that:

the $\mathrm{m} / \mathrm{n}$ ratio is consonant if and only if $\mathrm{m}$ and $\mathrm{n}$ are the number of sides of two regular polygons constructible with ruler and compass and $(m, n)=1$.

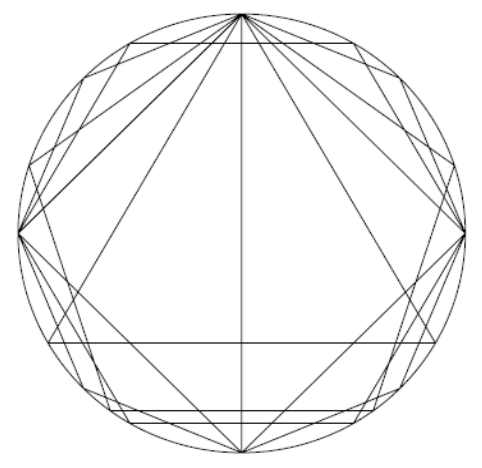

Figure 4: Kepler's musical scale.

Kepler used the music scale and made calculations based on it. He then provided the famous third law that governs the motion of the planets:

$$
T_{1}^{2}: T_{2}^{2}=r_{1}^{3}: r_{2}^{3}
$$


where $T_{1}$ and $T_{2}$ are periodic times and $r_{1}$ and $r_{2}$ are the main distance of the two planets, with $r_{2}>r_{l}$.

The model outlined in Mysterium Cosmographicum is maintained in the Harmonice mundi even though he added the speed of the planets at perihelion (minimum distance from the Sun) and at aphelion (maximum distance from the Sun) and melodies for the planets [11].

Saturn is the slower planet, thus it he occupies the lowest position in the musical scale: linking the $G$ to Saturn at aphelion, Kepler obtained the zarliniana scale, linking the $G$ to Saturn at perihelion he obtained the minor scale.

Each planet, however, can be represented with an interval whose extremes are the notes corresponding to its speed at aphelion and at perihelion.

Table 1: $\quad$ Kepler's scale.

\begin{tabular}{|c|c|c|}
\hline polygon & ratio & interval \\
\hline hexagon & $5 / 6$ & minor third \\
\hline pentagon & $4 / 5$ & major third \\
\hline square & $3 / 4$ & fourth \\
\hline triangle & $2 / 3$ & fifth \\
\hline octagon & $5 / 8$ & minor sixth \\
\hline pentagon & $3 / 5$ & major sixth \\
\hline diameter & $1 / 2$ & octave \\
\hline
\end{tabular}

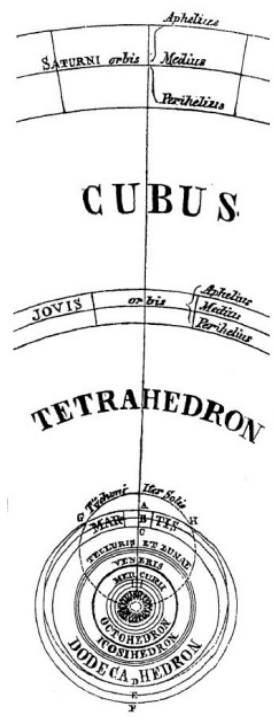

Figure 5: $\quad$ Model of the solar system in the Harmonice mundi. 

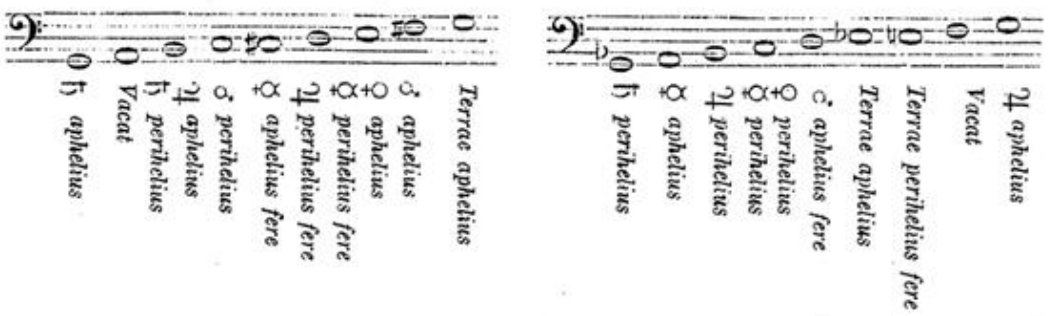

Figure 6: Zarlinian and minor scales obtained giving $\mathrm{G}$ to Saturn.

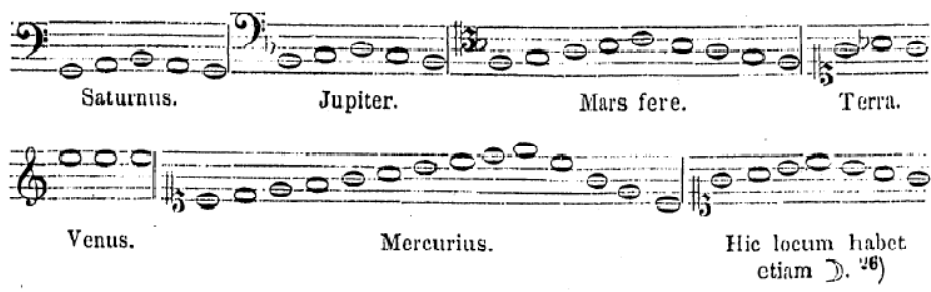

Figure 7: $\quad$ Planet's melodies in Harmonice mundi.

\section{Kepler, Galilei and Newton}

The Italian physicist and astronomer Galileo Galilei and the German astronomy Kepler started the scientific revolution that brought to the work of the English mathematician and physicist Isaac Newton [3, 10, 12].

Both Kepler and Galilei aimed to draw the design of the cosmos but they had different points of view and never worked together, as Kepler would liked to. Kepler focused on calculations and always tried to prove the elements of his "theory", while Galilei made physical experimentation. Although both supported the validity of the Copernican theory, the two astronomers never found an agreement. For example, dealing with tides: Kepler, in Astronomia nova, explained this phenomenon as an effect of attraction between the Earth and the Moon; Galileo explained it making assumptions about water motion and fluid dynamics. Nevertheless, both had an element in common, music: in particular, Galilei, that has received a complete musical education by his father Vincent, was the first to set the acoustics in scientific terms. He argued that the pitch derives, not only from the frequency of vibration of the string but also from the frequency of vibrations of the air. As his father said, even if the harmony of sounds follows the mathematical laws of Pythagoras, it derives from the natural vibrations of the air.

Newton, along with Copernicus, Galileo and Kepler, is considered one of the fathers of modern science. He also tried to describe the design of nature [7, 10]. Laws by Galileo and Kepler were taken into account by Newton for the description of physical phenomena: Galileo's studies on gravitation, which are 
the key to understand the three laws of dynamics, with the Kepler's laws, drove him to the definition of the law of universal gravitation.

Newton studied the system Sun-planet and considered the planet's motion as a uniform circular motion. Since the planet, that has period $\mathrm{T}$ and distance $r$ from the Sun, has an angular velocity $\omega=\frac{2 \pi}{T}$ and a centripetal acceleration $a_{c}=\omega^{2} r$, Newton's Second Law states that

$$
\vec{F}=m \vec{a}
$$

then he obtained that

$$
F=m \omega^{2} r=m \frac{4 \pi^{2}}{T^{2}} r .
$$

From Kepler's third law:

$$
T^{2}=k r^{3}
$$

thus Newton calculated that the force exerted on the planets from the sun is

$$
F_{S, P}=\frac{4 \pi^{2}}{k_{P}} \frac{m_{P}}{r^{2}}
$$

For the principle of action and reaction, the planet attracts the sun with a force of equal intensity and proportional to the mass of the Sun. Thus we have:

$$
F_{P, S}=\frac{4 \pi^{2}}{k_{S}} \frac{m_{S}}{r^{2}}
$$

Since $m_{P} k_{S}=m_{S} k_{P}$, Newton defined the gravitational constant:

$$
G=\frac{4 \pi^{2}}{m_{P} k_{S}}=\frac{4 \pi^{2}}{m_{S} k_{P}}
$$

and obtained the Law

$$
F=G \frac{m_{S} m_{P}}{r^{2}}
$$

Newton's method was based on geometry and on what he called the "method of fluxions" (the calculus). Nevertheless, he argued that his model of the cosmos could be explained also in musical terms. He wrote some scoli to the Principia Mathematica in which discusses the musical scale and the placement of the planets: his theory thus unified the motion of the planets with the acoustics trying to apply the gravitational law to the tension of the strings. 


\section{Conclusion}

Nature has been observed not only by physicists, mathematicians and scientists in general but also by musicians. Many musical works are the result of the observation of nature and natural phenomena, from the flight of birds to the flowing of water, from the arrangement of the stars to the colors of the seasons. Moreover, music is also relevant for our interpretation of the design of nature. The greatest scientists, such as Pythagoras, Kepler, Galelei and Newton have always referred to music to get the enunciation of their laws and the original mathematical model of the universe. Dealing with the relationship between music and the origin of the main theories on the universe in the history of science, we discussed the importance of music as an important instrument to understand the design of nature.

\section{References}

[1] Aiton, E.J., Duncan, A.M., Field, J.V., The harmony of the world, American Philosophical Society, Philadelphia 1997.

[2] Fauvel, J., Flood, R., Wilson, R., Music and Mathematics, from Pythagoras to Fractals, Oxford University Press, 2006.

[3] Gozza, P., La musica nella Rivoluzione Scientifica del Seicento, il Mulino, Bologna 1989.

[4] Grout, D.J., Storia della musica in Occidente, Feltrinelli, 2002.

[5] Kepler, J. Harmonice mundi libri V, 1619

[6] Kepler, J., Prodromus dissertationum cosmographicarum, continens Mysterium cosmographicum, Francoforte 1621.

[7] Koyré, A., La rivoluzione astronomica, Feltrinelli, 1966.

[8] Lombardi, A.M., Keplero, una biografia scientifica, Codice Edizioni, Torino 2008.

[9] Lozza, G., (eds). Platone, Timeo, Oscar Mondadori, 2006.

[10] Mamone Capria, M., (eds). La costruzione dell'immagine scientifica del mondo, Città del Sole, Napoli 1999.

[11] Morandi, F., Matematica e musica nell'Harmonice mundi di Keplero, Brigante Editore, 2010.

[12] Tonietti, T.M., Is music relevant for the history of the science? In The Application of Mathematics to the Sciences of Nature, edited by Verrai et al., Kluwer Academic/Plenum Publishers, New York, 2002. 